\title{
Evaluation of the Immunoregulatory Capacities of Feed Microbial Materials in Porcine Intestinal Immune and Epithelial Cells
}

\author{
Naosuke Kumagae ${ }^{1,2 *}$, Julio Villena1,3*, Yohsuke Tomosada ${ }^{*}$, Hisakazu Kobayashi', Paulraj \\ Kanmani',4, Hisashi Aso5, Takashi Sasaki'2, Motohiko Yoshida², Hiroshi Tanabe ${ }^{2}$, \\ Isao Shibata ${ }^{2}$, Tadao Saito ${ }^{1}$, Haruki Kitazawa ${ }^{1 \#}$ \\ ${ }^{1}$ Food and Feed Immunology Group, Tohoku University, Sendai, Japan \\ ${ }^{2}$ Scientific Feed Laboratory Co. Ltd., Tokyo, Japan \\ ${ }^{3}$ Laboratory of Immunobiotechnology, Reference Centre for Lactobacilli (CERELA-CONICET), \\ Tucuman, Argentina \\ ${ }^{4}$ Japan Society for the Promotion of Science (JSPS), Tokyo, Japan \\ ${ }^{5}$ Cell Biology Laboratory, Graduate School of Agricultural Science, Tohoku University, Sendai, Japan \\ Email: "
}

Received 9 February 2014; revised 9 March 2014; accepted 16 March 2014

Copyright (C) 2014 by authors and Scientific Research Publishing Inc.

This work is licensed under the Creative Commons Attribution International License (CC BY).

http://creativecommons.org/licenses/by/4.0/

(c) (i) Open Access

\section{Abstract}

The establishment of drug-free feeding systems has been required for secure and healthy livestock production. Although functional feed materials containing microorganisms as alternatives to enhance intestinal immunity are expected to be beneficial for reducing diarrhoea caused by pathogens in weaned piglets, the effects of such materials on porcine intestinal cells have not been investigated in detail. Therefore, this work evaluated the immunoregulatory functions of microbial feed materials in porcine intestinal immune and epithelial cells. Porcine immune cells isolated from Peyer's patches and mesenteric lymph nodes were stimulated with six different feed materials containing microorganisms, and evaluated for lymphocyte mitogenicity and cytokine inductions. In addition, porcine intestinal epithelial cells were stimulated with the materials before treatment with heat-killed enterotoxigenic Escherichia coli (ETEC), and analyzed for the proinflammatory cytokine expressions. The material containing Bifidobacterium thermophilum significantly augmented lymphocytes' mitogenicity and also induced a high expression of IL-2, IL-6 and IFN- $\gamma$ in immune cells, and inhibited ETEC-induced overexpression of IL-6 and IL-8 via regulation

\footnotetext{
*Theses authors have equal contribution.

\#Corresponding author.

How to cite this paper: Kumagae, N., Villena, J., Tomosada, Y., Kobayashi, H., Kanmani, P., Aso, H., Sasaki, T., Yoshida, M., Tanabe, H., Shibata, I., Saito, T. and Kitazawa, H. (2014) Evaluation of the Immunoregulatory Capacities of Feed Microbial Materials in Porcine Intestinal Immune and Epithelial Cells. Open Journal of Veterinary Medicine, 4, 15-28.

http://dx.doi.org/10.4236/ojvm.2014.43003
} 
of Toll-like receptor signaling. These results suggest that this feed material stimulates intestinal epithelial and immune cells to exert immunoregulation, suggesting that this feed is expected to contribute to promoting the health of piglets without using antimicrobial feed materials.

\author{
Keywords \\ Feed Microbial Material; Immunoregulatory Effect; Porcine Intestinal Epitheliocytes; \\ Porcine Immune Cells
}

\title{
1. Introduction
}

Weaning-associated intestinal inflammation occurs in various animal species including the pig. Following the withdrawal of sow's milk, young piglets are highly susceptible to enteric diseases partly as a result of the altered balance between developing beneficial microbiota and the establishment of intestinal bacterial pathogens. In addition to the changes in microbiota composition, the intestinal immune system of the newborn piglet undergoes a rapid period of maturation, expansion, and specialization that is not achieved before commercial weaning [1]. Then, intensification of the pig industry has brought increased risks of both clinical and sub-clinical enteric disease. Piglets are vulnerable to harmful microorganisms such as Escherichia coli, Salmonella spp., and Clostridium perfringens [2]. Antibiotics have been applied widely in animal husbandry to prevent and treat the gastrointestinal infection caused by pathogens [3]. However, the promiscuous use of antibiotics has resulted not only in the emergence and spread of resistant bacteria in humans but also in animals [4].

Various nutritional approaches for optimizing the weaning transition and minimizing gut inflammation and enteric diseases have been tested in the past decade. Among the novel dietary strategies investigated that are focused on improving gut health in pigs, probiotics and prebiotics are clear nutritional options. A growing body of evidence supports the therapeutic and preventive application of probiotics for several gastrointestinal disorders in pigs. Qiao et al. [5] conducted experiments to evaluate the effects of a complex Lactobacilli preparation on performance, resistance to $E$. coli infection and gut microbial flora of weaning pigs. The mix of four lactobacilli isolated from weaning pigs was able to reduce $E$. coli and anaerobe counts in the gut, and decrease diarrhoea. Additionally, lactobacilli treatment significantly improved average daily feed intake of pigs [5]. Another study from Herfel et al., [6] examined the impact of a novel probiotic strain of Bifidobacterium longum AH1206 on the health, growth and development of neonatal pigs. Authors found that ileal IL-10 expression increased progressively with AH1206 supplementation, which indicated the potential for modulation of the inflammatory tone of the intestinal mucosa of suckling piglets. However, no differences were found between AH1206-treated and control piglets when comparing body weight gain, feed efficiency (gain:intake), and physiological modifications in the gut. Another recent study evaluated the effect of the co-administration of Bacillus subtilis RJGP16 and Lactobacillus salivarius B1 on intestinal immunity in piglets [7]. Authors demonstrated that probiotic administration increased the expression of IL-6, porcine beta-defensins and IgA producing cells in the intestine, clearly showing that co-administration of RJGP16 and B1 strains strongly enhances the intestinal mucosal immunity of piglets.

In addition, some recent studies have specifically evaluated the capacity of probiotics to improve the resistance of piglets against enterotoxigenic Escherichia coli (ETEC). It was shown that the probiotic strain L. plantarum CJLP243 may serve as a potential alternative to antibiotic supplementation to improve the growth and health performance of weaning pigs because of its capacity to reduce the severity of ETEC-induced diarrhoea [8]. Li et al., [9] showed that pretreatment of piglets with L. rhamnosus ATCC7469 ameliorates F4 ${ }^{+}$ETEC-induced diarrhoea. In piglets exposed to F4 ${ }^{+}$ETEC, jejunal Toll-like receptor (TLR) 4 and IL-8 expression were increased; however, these increases were attenuated by administration of L. rhamnosus. Notably, expression of jejunal TLR2, ileal TLR9, NOD1 and TNF- $\alpha$ was upregulated in the ATCC7469-treated piglets after F4 ETEC challenge [9].

Although these studies demonstrated that it is possible to modulate piglets' gut microbiota and immunity resulting in improvement of growth performance by using appropriate probiotics strains, the true efficacy of probiotics in livestock animals remains unclear because of incomplete studies of mechanisms. In this regard, some feed microbial materials (FMAs) containing Aspergilli, Lactobacilli and Bifidobacteria have been used to successfully improve growth performance in livestock in Japan [10]. However, the exact cellular and molecular 
mechanisms involved in the beneficial effects have not been studied in detail.

Our laboratory has shown that probiotic microorganism with immunoregulatory functions (immunobiotics) can beneficially modulate the immune response in the gut by modulating the functions of intestinal epithelial cells (IECs) and immune cells [11]-[15]. Therefore, the aims of the present work were: 1) to evaluate the immunoregulatory capacities of common microbial feed materials used in Japan in porcine intestinal immune and epithelial cells and; 2) to advance in the knowledge of the cellular and molecular mechanisms involved in the immunoregulatory effect these materials by evaluating the role of TLRs and negative regulators of TLR.

\section{Materials and Methods}

\subsection{Feed Microbial Materials}

The following feed microbial materials (FMAs) used as livestock feed in Japan were evaluated in this study. FMA1: Grinded freeze dry soymeal fermented with Enterococcus faecium and Aspergillus oryzae. FMA2: Freeze dry of Candida utilis cultures. FMA3: Freeze dry of Saccharomyces cerevisiae cultures. FMA4: Freeze dry of Enterococcus faecalis cultures. FMA5: Freeze dry of Bifidobacterium thermophilum cultures. FMA6: Freeze dry of Leuconostoc mesenteroides cultures.

Enterotoxigenic Echerichia coli (ETEC) strain 987P was kindly provided by the National Institute of Animal Health (Tsukuba, Japan). ETEC cells were plated into tryptic soy agar (TSA, Becton, Dickinson and Company) supplemented with 5\% sheep blood (Nippon Biotest Laboratories Inc., Tokyo, Japan). After overnight incubation at $37^{\circ} \mathrm{C}$, ETEC was transferred to tryptic soy broth (TSB, Becton) and grown for 5 days without shaking to form a pellicle containing the piliated phase. Then, ETEC cells were collected from the pellicle and transferred to TSB and cultured 20 hours at $37^{\circ} \mathrm{C}$ with shaking (200 rpm). Finally, the subculture of the ETEC strain was centrifuged at $1900 \times \mathrm{g}$ for $10 \mathrm{~min}$ at $4^{\circ} \mathrm{C}$, washed with phosphate buffered saline (PBS) and heat-killed at $65^{\circ} \mathrm{C}$ for $30 \mathrm{~min}$, and resuspended in Dulbecco's modified Eagle’s medium (DMEM) (Invitrogen corporation, Carlsbad, CA) as described previously [12] [13].

\subsection{Porcine Intestinal Epitheliocyte (PIE) Cells}

Porcine intestinal epitheliocyte (PIE) cells were obtained as described before [16]. PIE cells were maintained in DMEM (Invitrogen Corporation, Carlsbad, CA) supplemented with 10\% fetal calf serum (FCS), $100 \mathrm{mg} / \mathrm{ml}$ penicillin, and $100 \mathrm{U} / \mathrm{ml}$ streptomycin at $37^{\circ} \mathrm{C}$ in an atmosphere of $5 \% \mathrm{CO}_{2}$. PIE cells grow rapidly and are well adapted to culture conditions even without transformation or immortalization. However, the proliferative ability of PIE cells diminishes after 50 passages in culture. Therefore, we used PIE cells only between the 20th and 40th passages in experiments.

\subsection{Immunocompetent Cells from Swine Peyer's Patches and Mesenteric Lymph Nodes}

This study was carried out in strict accordance with the recommendations in the Guide for the Care and Use of Laboratory Animals of the Guidelines for Animal Experimentation of Tohoku University, Sendai, Japan. The present study was approved by the Institution Animal Care and Use Committee of Tohoku University with a permitted No. 2011-noudou-5. All efforts were made to minimize suffering. Immunocompetent cells from Swine peyer's patches (PPs) and mesenteric lymph nodes (MLNs) were prepared from adult swine intestine as described previously [11] [14]. Briefly, PPs or MLNs were cut into small fragments, which were gently pressed through a nylon mesh and washed three times in complete RPMI 1640 medium (Sigma, St. Louis, MO) supplemented with 10\% FCS (Sigma). Residual erythrocytes were lysed by resuspending in hypotonic salt solution $(0.2 \% \mathrm{NaCl})$. Next, harvested PPs or MLN cells were subjected to hypertonic rescue in an equal volume of $1.5 \%$ $\mathrm{NaCl}$. Finally, immune cells were fractionated using Lympholyte-mammal (Cedarlane, Hornby, Ontario, Canada) density gradient centrifugation, and the isolated immune cells were suspended in complete DMEM (Invitrogen, Tokyo, Japan) supplemented with 10\% FCS (Sigma) and 50 mg/ml penicillin-streptomycin (Life Tech., Carlsbad, CA, USA).

\subsection{Mitogenicity Assay}

The mitogenicity assay was performed as described previously [11]. Briefly, PPs or MLN immunocompetent 
cells were placed in a 96-well microplate (Costar, $2 \times 10^{5}$ cells/well) and incubated at $5 \% \mathrm{CO}_{2}, 37^{\circ} \mathrm{C}$ for $48 \mathrm{~h}$ in complete RPMI 1640 medium (Sigma) supplemented with 2\% FCS. Immune cells were stimulated with FMAs $(0.1-1000 \mu \mathrm{g} / \mathrm{mL})$ for $48 \mathrm{~h}$. During the final hour of culture, the cells were radiolabeled with $9.25 \mathrm{kBq}$ per well of [methyl- $\left.{ }^{3} \mathrm{H}\right]$-Thymidine (GE Healthcare, Tokyo, Japan). The cells were then harvested with a glass fibre filter (PerkinElmer Japan, Kanagawa, Japan). The [methyl- $\left.{ }^{3} \mathrm{H}\right]$-Thymidine incorporation was quantitated in a liquid scintillation counter (Beckman Instruments, Palo Alto, CA, USA). Results are presented as the Stimulation Index (SI), calculated with the following equation:

$$
\frac{[(\text { counts per minute in treated cultures })-(\text { counts per minute in background })]}{[(\text { counts per minute in control cultures })-(\text { counts per minute in background })]}[11] \text {. }
$$

\subsection{Immunomoregulatory Effect of FMAs in Immune Cells and PIE Cells}

Evaluation of the immunoregulatory activity of FMAs was performed using mononuclear cells from PPs and MLNs or PIE cells. PP and MLM cells ware plated at a density of $4.0 \times 10^{6}$ cells/well in 12-well type I collagencoated plates (Iwaki, Tokyo, Japan). PIE cells were plated at a density of $3.0 \times 10^{4}$ cells/well in 12-well type I collagen-coated plates (Iwaki) and cultured for 3 days before stimulation of FAMs. FMAs were added into each well and cells were incubated for 48 h. Expression of IFN- $\gamma$, IL-2, IL-4, IL-6, IL-8, IL-10, IL-13 and MCP-1 was evaluated with quantitative real time RT-PCR as described below. In addition, expression levels of six TLR negative regulators, A20, Bcl-3, IRAK-M, MKP-1, SIGIRR, and Tollip, were evaluated in PIE cells as described previously [12] [13].

\subsection{Anti-Inflammatory Effect of FMAs in PIE Cells}

PIE cells were seeded at $3 \times 10^{4}$ cells/12-well plate on type I collagen-coated plates (Iwaki) and cultured for 3 days. After changing medium, FMAs were added; $48 \mathrm{~h}$ later, each well was washed vigorously with medium at least three times to eliminate all stimulants, and then cells were stimulated with ETEC (equivalent to $5 \times 10^{7}$ cells $/ \mathrm{ml}$ ) for $12 \mathrm{~h}$. Expression of cytokines was evaluated with quantitative real time RT-PCR as described below. In blocking experiments, unlabeled anti-porcine TLR2- or TLR4-rabbit IgG (Biolegend, San Diego, CA) were used. Cultured porcine cells were incubated with unlabeled anti-TLR2 of TLR4 antibodies for $12 \mathrm{~h}$ before stimulation with FMAs.

\subsection{Real-Time Quantitative RT-PCR}

We performed two-step real-time quantitative PCR to characterize the expression of mRNAs in PIE cells and immune cells. Total RNA was isolated from each PIE or immune cell sample using TRIzol reagent (Invitrogen). All cDNAs were synthesized using a Quantitect reverse transcription (RT) kit (Qiagen, Tokyo, Japan) according to the manufacturer's recommendations. Real-time quantitative PCR was carried out using a 7300 real-time PCR system (Applied Biosystems, Warrington, UK) and the Platinum SYBR green qPCR SuperMix uracil-DNA glycosylase with 6-carboxyl-X-rhodamine (Invitrogen). The primers for IFN- $\gamma$, IL-2, IL-4, IL-6, IL-8, IL-10, IL13, MCP-1, A20, SIGIRR, Tollip, Bcl-3, MKP-1, and IRAK-M used in this study were previously described [11] [12]. The PCR cycling conditions were $5 \mathrm{~min}$ at $50^{\circ} \mathrm{C}$, followed by $5 \mathrm{~min}$ at $95^{\circ} \mathrm{C}$, and then 40 cycles of $15 \mathrm{~s}$ at $95^{\circ} \mathrm{C}, 30 \mathrm{~s}$ at $60^{\circ} \mathrm{C}$, and $30 \mathrm{~s}$ at $72^{\circ} \mathrm{C}$. The reaction mixtures contained $2.5 \mathrm{~m} / \mathrm{l}$ sample cDNA and $7.5 \mathrm{~m} / \mathrm{l}$ master mix, which included the sense and antisense primers. Expression of $\beta$-actin in each sample was assessed, and the $\beta$-actin data were used as an internal control to normalize differences between samples and to calculate normalized fold expression.

\subsection{Western Blot Analysis}

PIE cells were resuspended in $200 \mu$ l CelLytic M cell lysis reagent (Sigma) including protease and phosphate inhibitors (Complete Mini, PhosSTOP; Roche, Mannheim, Germany). Cells were transferred into Eppendorf tubes and boiled for $5 \mathrm{~min}$ at $95^{\circ} \mathrm{C}$. Protein concentration was measured using the bicinchoninic acid protein assay kit (Pierce, Rockford, IL). Total protein samples ( $2 \mu \mathrm{g} /$ sample) were loaded onto 10\% SDS-polyacrylamide gels. Separated proteins were electrophoretically transferred to a nitrocellulose membrane. Phosphorylation of 
$\mathrm{p} 38$, and IкB $\alpha$ degradation were evaluated using anti-phosphorylated $\mathrm{p} 38$, and anti-I $\kappa \mathrm{B}$ antibodies, respectively (Cell Signaling Technology, Beverly, MA). After detecting the phosphorylation, the membranes were stripped with Ten Minute Western Blot Re-Probe Kit (Jacksun Easy Biotech, Inc., New York, USA) for the detection of each total protein using p38 antibody (Cell Signaling Technology) and -actin antibody (Cell Signaling Technology). Anti-rabbit IgG, AP-linked antibody (Cell Signaling Technology) was used as secondary antibody. The optical protein bands were detected by ECF substrate (GE Healthcare Japan Co., Tokyo, Japan) and estimated from the peak area of densitogram by using Image J software (National Institute of Health, Bethesda, MD, USA).

\subsection{Statistical Analysis}

Statistical analyses were performed using the GLM and REG procedures available in the SAS computer program (SAS, 1994). Comparisons between mean values were carried out using one-way analysis of variance and Fisher's least-significant-difference test. For these analyses, $P$ values of 0.05 were considered significant.

\section{Results}

\subsection{Immunoregulatory Activity of Feed Microbial Materials in Porcine Intestinal Immune Cells}

We first evaluated the mitogenic capacity of the feed microbial materials (FMAs) in porcine intestinal immune isolated from Peyer's patches (PPs) or MLN (Figure 1).

All the FMAs were able to significantly increase the mitogenic activity of PPs and MLN cells compared with the control group, in a dose dependent manner. However, FMA5 was the most effective treatment to increase this parameter in MLN cells, being the doses of $100 \mu \mathrm{g} / \mathrm{ml}$ and $1000 \mu \mathrm{g} / \mathrm{ml}$ able to increase mitogenic activity more than 10 folds (Figure 1(a)). In addition, both FMA4 and FMA5 significantly increased mitogenic activities of immune isolated from PPs, being FMA5 in a dose of $100 \mu \mathrm{g} / \mathrm{ml}$ the most effective treatment to increase this parameter (Figure 1(b)).

We also evaluated the changes in cytokine expression in immune isolated from PPs or MLN after the treatments with the FMAs. As shown in Figure 2(a), all the treatments were able to upregulate the expression of IL4 and IL-6 in MLN cells. In addition, FMA5 significantly increased the expression of IL-2, IL-10 and IL-13 in those cells (Figure 2(a)). On the other hand, changes in the expression of cytokines in immune isolated from PPs were different for each FMA (Figure 2(b)). All the FMAs upregulated the expression of IFN- $\gamma$, being FMA5 and FMA6 the most effective treatments for improving this cytokine. In addition, FMA5 effectively increased the expression of IL-2 and IL-6 in PPs cells while FMA6 significantly increased IL-4 and IL-13 (Figure 2(b)).

\subsection{Immunoregulatory Activity of Feed Microbial Materials in PIE Cells}

The immunoregulatory capacities of FMAs were then evaluated in PIE cells. First, PIE cell were stimulated with the different FMAs and the expression of IL-6, IL-8 and MCP-1 was evaluated (Figure 3(a)).

FMA5 significantly increased the expression of IL-6 in PIE cells while FMA6 upregulated the expression of MCP-1 (Figure 3(a)).

In addition, the effect of FMAs in the response of PIE cell to heat-killed ETEC challenge was evaluated. As shown in Figure 3(b), ETEC challenge significantly increased the production of proinflammatory cytokines in PIE cells. However, PIE cells pretreated with FMA5 before the challenge with ETEC showed significantly lower levels of IL-6, IL-8 and MCP-1 when compared to ETEC controls (Figure 3(b)). In addition, both FMA4 and FMA6 were able to reduce IL-8 expression.

We next evaluated whether FMA5 attenuated the ETEC-mediated proinflammatory response by modulating the NF- $\kappa$ B pathway (Figure 4(a)). Challenge of PIE cells with ETEC significantly reduced the levels of the counter-regulatory factor $\mathrm{I} \kappa \mathrm{B} \alpha$ after 5 to $40 \mathrm{~min}$, indicating activation of the NF- $\kappa \mathrm{B}$ pathway. PIE cells previously stimulated with FMA5 or FMA1 showed significantly higher levels of I $\mathrm{KB} \alpha$ when compared to control cells. In fact, no significant degradation of $\mathrm{I} \kappa \mathrm{B} \alpha$ was observed in FMAs-treated PIE cells, indicating an inhibitory effect of the NF- $\kappa$ B pathway (Figure 4(a)). We also examined the relationship between p38 MAPK activa- 


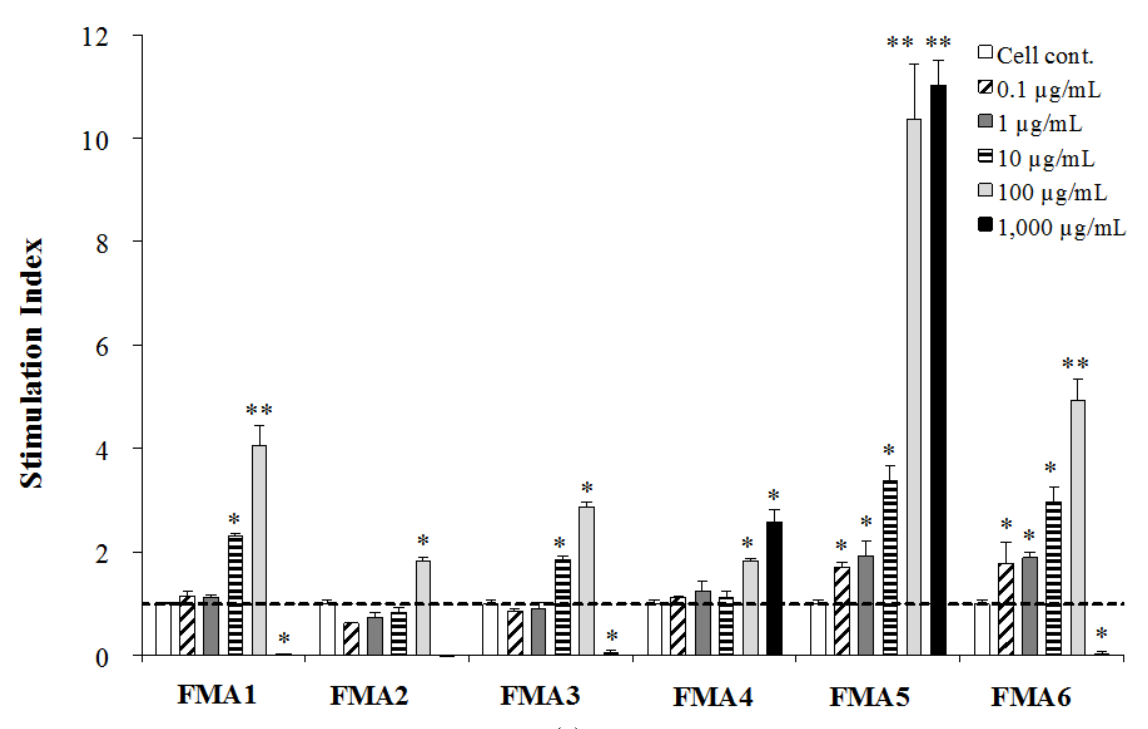

(a)

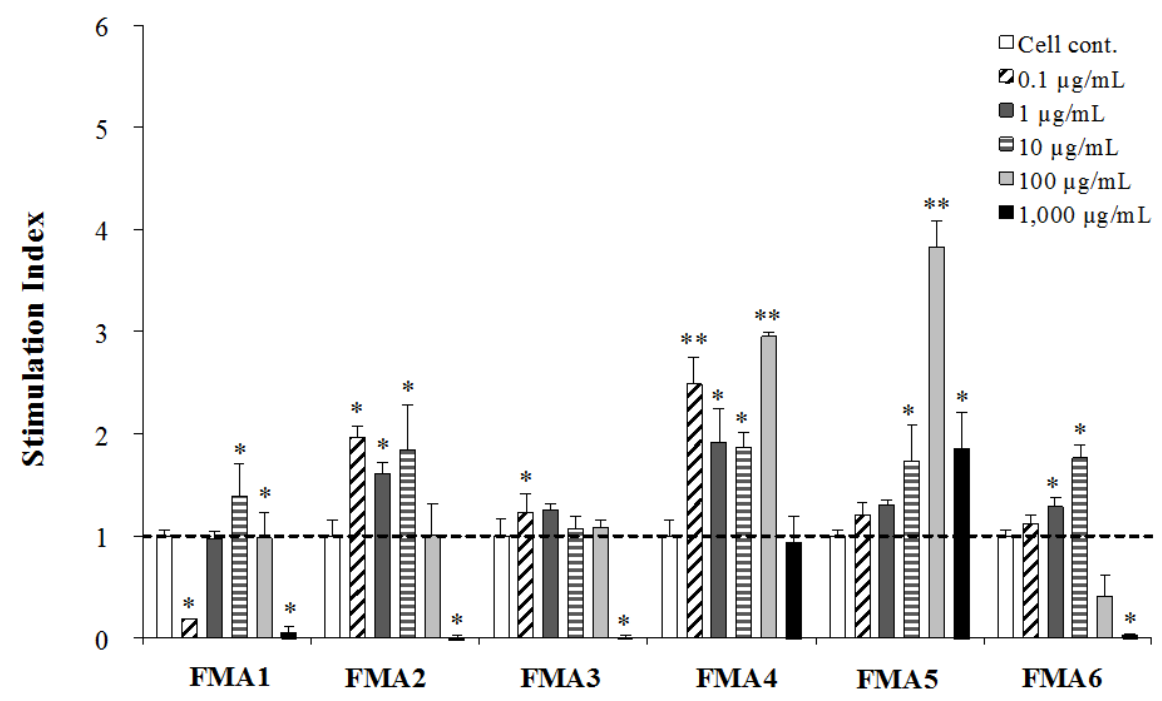

(b)

Figure 1. Mitogenic activity of functional feed materials (FMAs) on porcine mesenteric lymphoid nodes (MLN) (a) and Peyer's patches (PPs) (b) cells. FMA1: Grinded freeze dry soymeal fermented with Enterococcus faecium and Aspergillus oryzae. FMA2: Freeze dry of Candida utilis cultures. FMA3: Freeze dry of Saccharomyces cerevisiae cultures. FMA4: Freeze dry of Enterococcus faecalis cultures. FMA5: Freeze dry of Bifidobacterium thermophilum cultures. FMA6: Freeze dry of Leuconostoc mesenteroides cultures. Values represent means and error bars indicate the S.D. The results are representative of three independent experiments. ${ }^{*} \mathrm{p}<0.05,{ }^{* *} \mathrm{p}<0.01$ against cell control.

tion and the regulation of proinflammatory cytokines in PIE cells by FMA5 (Figure 4(b)). PIE cells were stimulated with FMA1, FMA5, or control medium, and the activation profiles of p38 after ETEC challenge was compared. ETEC challenge induced the phosphorylation of p38 after 5 min. FMA5-treated PIE cells showed significantly lower levels of phosphorylated p38 than control cells (Figure 4(b)).

\subsection{Effect of Feed Microbial Materials on Negative Regulators of the TLR Signaling Pathway in PIE Cells}

We also studied regulators that inhibit the TLR signaling pathway. The expression of SIGIRR, Tollip, A20, Bcl- 


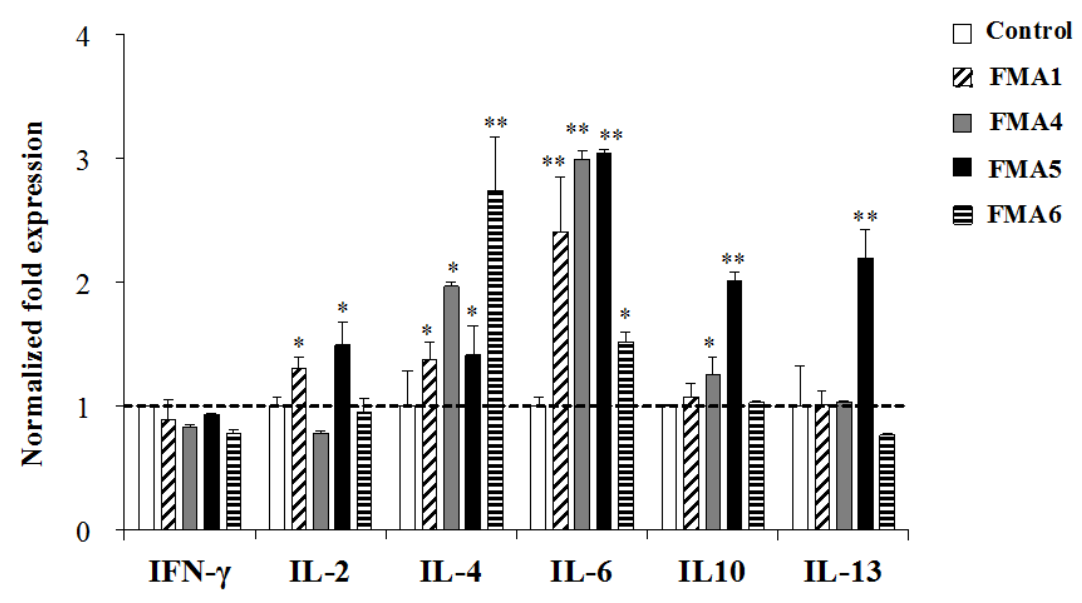

(a)

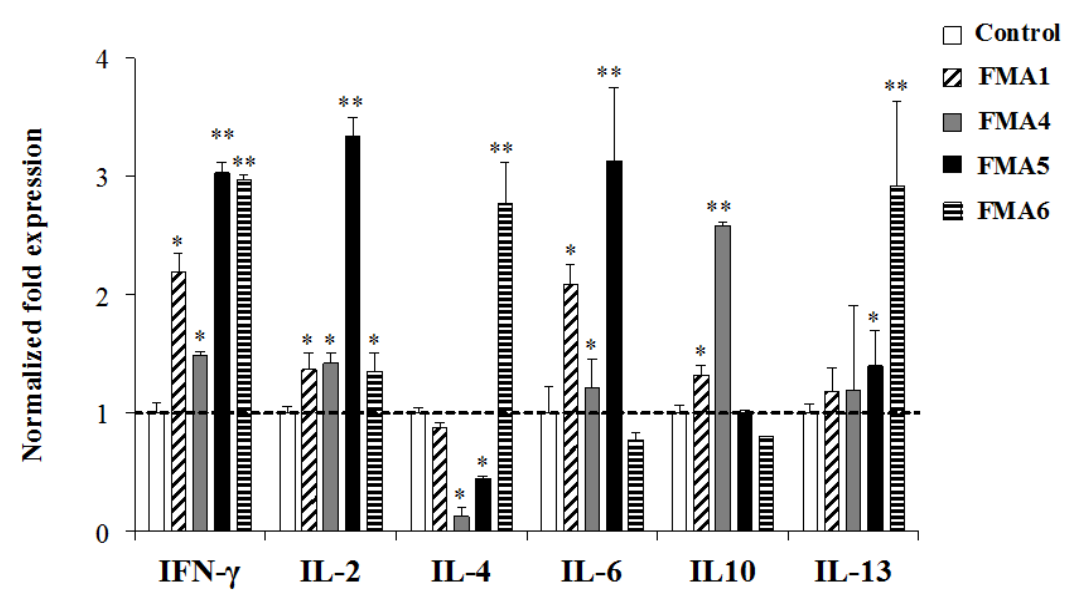

(b)

Figure 2. Expression of cytokines mRNA in porcine mesenteric lymphoid nodes (MLN) (a) and Peyer's patches (PPs) (b) cells stimulated with functional feed materials (FMAs). FMA1: Grinded freeze dry soymeal fermented with Enterococcus faecium and Aspergillus oryzae. FMA4: Freeze dry of Enterococcus faecalis cultures. FMA5: Freeze dry of Bifidobacterium thermophilum cultures. FMA6: Freeze dry of Leuconostoc mesenteroides cultures. Values represent means and error bars indicate the S.D. The results are representative of three independent experiments. ${ }^{*} \mathrm{p}<0.05,{ }^{* *} \mathrm{p}<0.01$ against control.

3, MKP-1, and IRAK-M mRNAs in PIE cells was determined after the challenge with heat-killed ETEC (Figure 5). None of the treatments induced statistical significant changes in expression of Bcl-3 (Figure 5). However, all FMAs except FMA6 caused upregulation of SIGIRR. Additionally, FMA5 significantly increased the expression of Tollip and IRAK-M and A20 (Figure 5). FMA6 also upregulated A20 and IRAK-M while Bcl-3 was increased in PIE cells only with FMA1 (Figure 5).

\subsection{Role of TLRs in the Immunoregulatory Activity of Feed Microbial Materials}

To study the role of TLR2 and TLR4 in the immunomodulatory effect of FMA5, we next performed comparative studies with FMA5 and FMA1 and used anti-TLR2 and anti-TLR4 blocking antibodies, in PIE cells challenged with heat-killed ETEC (Figure 6). The use of anti-TLR2 antibodies did not induce any modification in the capacity of FMA5 to downregulate the expression of IL-6 or IL-8 in ETEC-challenged PIE cells, while the reduction of MCP-1 was abolished with anti-TLR2 antibodies (Figure 6). On the contrary, reduction of IL-6 and IL-8 induced by FMA5 was abolished with anti-TLR4 antibodies, while these blocking antibodies did not induce changes in the capacity of FMA5 to reduce MCP-1 (Figure 6). 


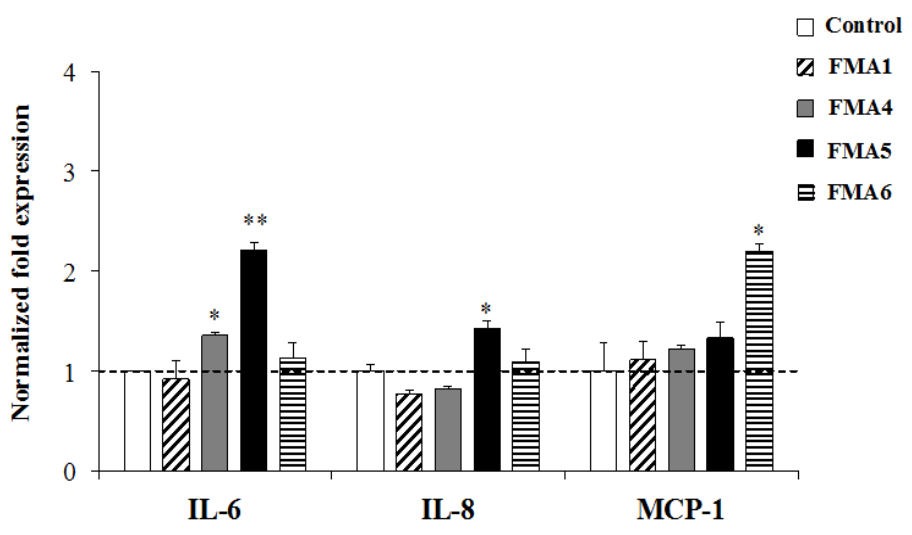

(a)

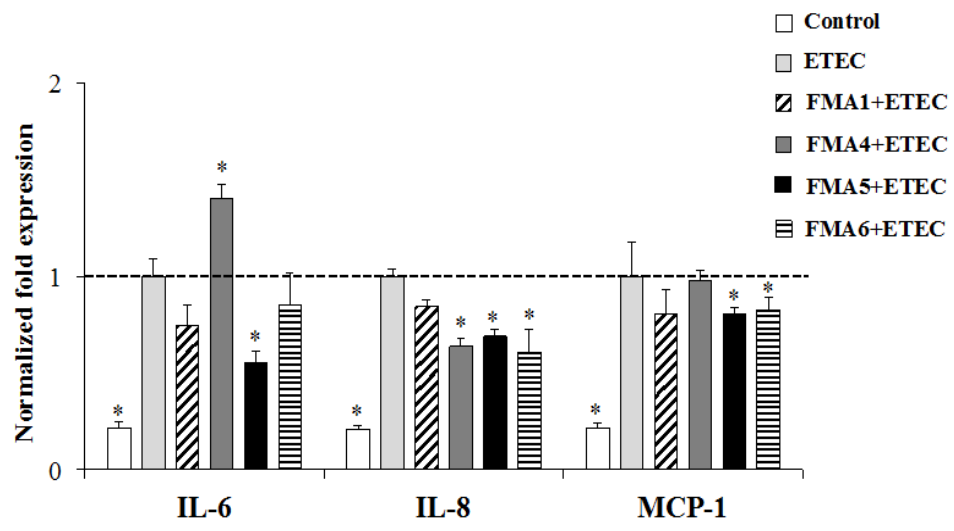

(b)

Figure 3. Effect of feed microbial materials (FMAs) on the expression of IL-6, IL-8 and MCP-1 mRNA in porcine intestinal epithelial (PIE) cells in non inflammatory conditions (a) and after inflammatory stimulation (b). (a) PIE cells were pre-treated with FMAs for $48 \mathrm{~h}$ or, (b) stimulated with FMAs for $48 \mathrm{~h}$ and then stimulated with heat-killed enterotoxigenic Escherichia coli (ETEC) for $12 \mathrm{~h}$. PIE cells without pre-treatment (ETEC control group) and without ETEC challenge (control group) were used as controls. FMA1: Grinded freeze dry soymeal fermented with Enterococcus faecium and Aspergillus oryzae. FMA4: Freeze dry of Enterococcus faecalis cultures. FMA5: Freeze dry of Bifidobacterium thermophilum cultures. FMA6: Freeze dry of Leuconostoc mesenteroides cultures. Values represent means and error bars indicate the S.D. The results are representative of four independent experiments. ${ }^{*} \mathrm{p}<0.05,{ }^{* *} \mathrm{p}<0.01$ against ETEC control.

\section{Discussion}

The weaning transition is a complex period during which the piglets have to face abrupt changes. They are separated from their mother, mixed with other litters in a new environment, and switched from milk to a solid feed which involves a change from a highly digestible to a less-digestible and more-complex feed. In consequence, several physiological changes occur in the intestine of pigs during weaning which increase the susceptibility to uncontrolled inflammation and infections [reviewed in 17]. Probiotic microorganisms have been proposed to functionally modulate the microbiota and the immune system during the weaning transition in the pig, in order to avoid gut alterations and increase pig productivity. In Japan, several FMAs are used as expected alternatives to antibiotics to improve pig health. However, the exact cellular and molecular mechanisms involved in the beneficial effects obtained in pigs have not been studied in detail. In this work we evaluated the immunoregulatory capacities of several FMAs using porcine in vitro systems, and we found that the freeze dry of $B$. thermo- 

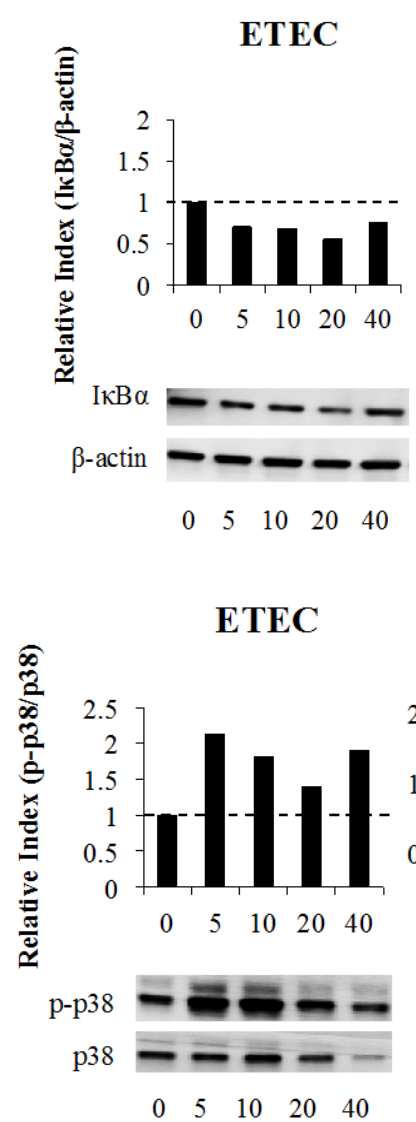

FMA1+ETEC
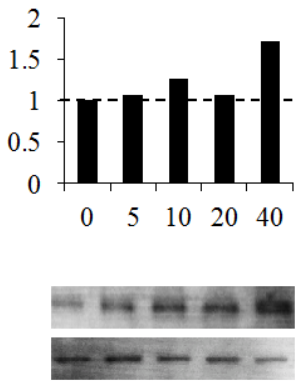

$\begin{array}{lllll}0 & 5 & 10 & 20 & 40\end{array}$

(a)

FMA1+ETEC

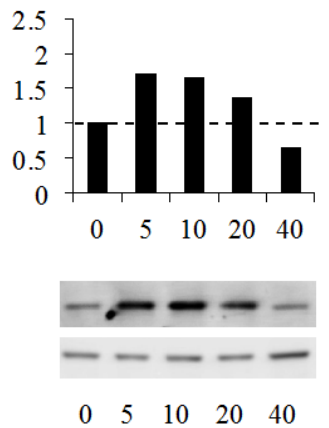

(b)
FMA5+ETEC

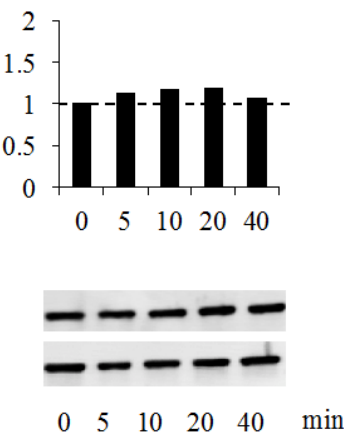

FMA5+ETEC
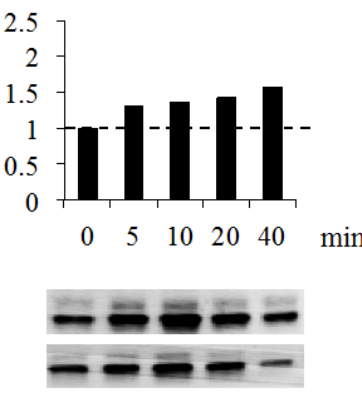

$\begin{array}{llllll}0 & 5 & 10 & 20 & 40 & \min \end{array}$

Figure 4. Effect of feed microbial materials (FMAs) on NF-B and p38 mitogen-activated protein kinase pathways in porcine intestinal epithelial (PIE) cells after inflammatory stimulation. PIE cells were pre-treated with FMAs for $48 \mathrm{~h}$ and then stimulated with heat-killed enterotoxigenic Escherichia coli (ETEC). The degradation of $\mathrm{I} \kappa \mathrm{B} \alpha$ and phosphorylation of p38 was evaluated at different time intervals $(0,5,10,20$, and 40 min). Intensities of protein bands were calculated from the peak area of densitogram by using image software and were expressed as bar graph of relative index with setting 1.0 at time 0. FMA1: Grinded freeze dry soymeal fermented with Enterococcus faecium and Aspergillus oryzae. FMA5: Freeze dry of Bifidobacterium thermophilum cultures.

philum cultures (called FMA5 in this work) was notably able to modulate porcine immune cells as well as PIE cells.

Mitogenic activities are generally considered to be one of the most important properties of immune modulators, and have been used to evaluate the immunomodulating activities of potential probiotic microorganisms. Here, we used this methodology to evaluate the immunoregulatory effect of different FMAs in porcine immune cells. Results showed that FMA5 significantly increased mitogenic activity of both PPs and MLN cells. Moreover, FMA5 was able to increase the expression of IL-2, IL-6, IL-10 and IFN- $\gamma$ in porcine immune cells indicating a clear immunostimulatory. In fact, early studies from Sasaki et al. [10] demonstrated that oral administration of $B$. thermophilum peptidoglycan to weaning piglets significantly improved intestinal humoral immunity and protected against post-weaning diarrhoea. Authors demonstrated that $B$. thermophilum peptidoglycan administration augments IgA+ cells in pigs' intestine, which correlates with the augmented levels of IL-6 and IL-10 found in our experiments. Moreover, we extend these findings by demonstrating the capacity of $B$. thermophilum to improve intestinal Th1 immunity. This capacity of FMA5 to improve IgA and to stimulate both IL-10 and IFN- $\gamma$ in immune cells could explain the beneficial effect in vivo because the administration of this FMA may result in an improvement in the adaptive immune response and protection from unproductive inflammation. In fact, evidence from in vivo trials of some strains indicates that immunobiotics can simultaneously 

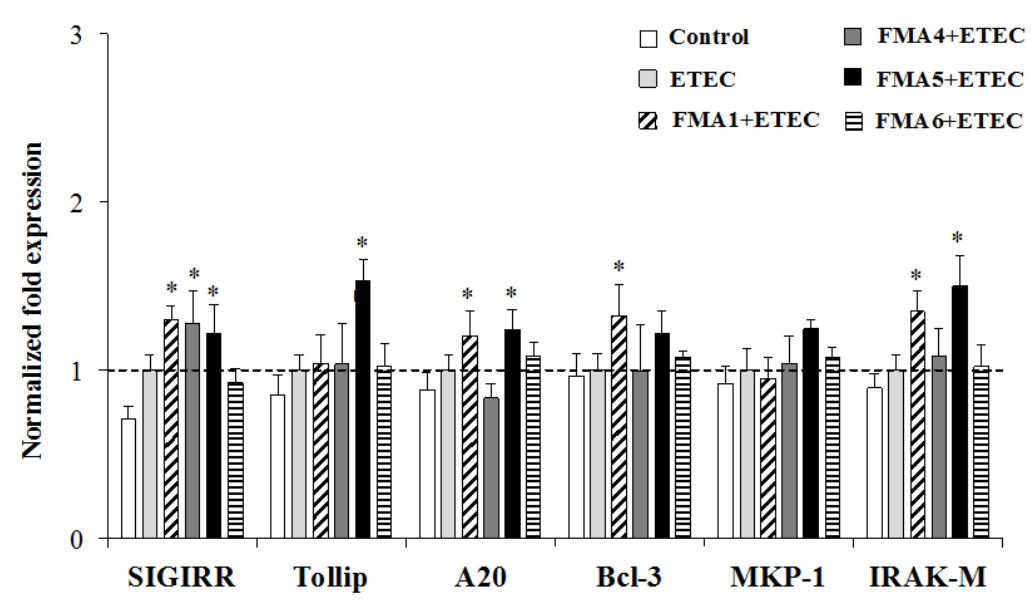

Figure 5. Effect of feed microbial materials (FMAs) on the expression of tolllike receptor negative regulators in porcine intestinal epithelial (PIE) cells. PIE cells were pre-treated with different FMAs for $48 \mathrm{~h}$, stimulated with heat-killed enter otoxigenic Escherichia coli (ETEC) for $3 \mathrm{~h}$, and the expression of SIGIRR, Tollip, A20, Bcl-3, MKP-1, and IRAK-M negative regulators was evaluated. FMA1: Grinded freeze dry soymeal fermented with Enterococcus faecium and Aspergillus oryzae. FMA4: Freeze dry of Enterococcus faecalis cultures. FMA5: Freeze dry of Bifidobacterium thermophilum cultures. FMA6: Freeze dry of Leuconostoc mesenteroides cultures. Values represent means and error bars indicate the S.D. The results are representative of three independent experiments. p $<0.05$ against ETEC control.

improve both resistance against infection and protection from inflammatory tissue damage [17]-[19].

In addition, we showed that FMA5 is able to functionally modulate PIE cells. Previously, we demonstrated that the stimulation of PIE cells with ETEC significantly increases the levels of IL-6, IL-8, and MCP-1 [12] [16]. It was also found that damage to PIE cells correlates with the levels of proinflammatory cytokines produced after stimulation with heat-killed ETEC and LPS [12], which is consistent with reports demonstrating that challenging human intestinal Caco-2 cells with ETEC causes strong up-regulation of proinflammatory mediators that lead to membrane damage [20] [21]. Then, this porcine in vitro system is a useful tool to evaluate the immunoprotective capacity of potential probiotic microorganisms. In this work, experiments with PIE cells allow us to conclude that FMA5 has an immunoprotective effect against ETEC challenge. The stimulation of PIE cells with ETEC significantly increased levels of proinflammatory cytokines in control cells. However, IL-6, IL-8 and MCP-1 levels in PIE cells stimulated with FMA5 were significantly lower than those in the control. Moreover we showed that this effect was related to the capacity of FMA5 to modulate NF-kB and p38 MAPK pathways in PIE cells. This is in line with reports demonstrating that probiotics can inhibit excessive NF-kB and MAPK induced by proinflammatory cytokine secreted from IECs. Immunobiotics can suppress TNF or Salmonella enterica serovar Typhimurium-induced IL-8 mRNA expression and secretion by IECs in an NF-kB-dependent manner [22]. Moreover, we demonstrated that some immunobiotic strains such as L. jensenii TL2937, B. longum BB536, B. breve M-16V and B. breve MCC-117 significantly downregulated levels of IL-8, MCP-1, and IL-6 in PIE cells challenged with ETEC by modulating the NF-kB and p38 MAPK pathways [12] [13] [16].

To dissect the mechanism(s) involved in the immunoregulatory effect of FMA5 we evaluated the expression of the negative TLR regulators in PIE cells. The expression of SIGIRR, Tollip, A20, Bcl-3, MKP-1, and IRAK$M$ was studied, and it was found that SIGIRR, Tollip, A20 and IRAK-M expression was upregulated in PIE cells stimulated with FMA5.

It was shown in vitro that overexpression of SIGIRR inhibits TLR-induced NF- $\kappa$ B activation and attenuates the production of inflammatory cytokines [23]. The LPS-induced inflammatory response is enhanced in SIGIRR-deficient mice [24]. Notably, IRAK-M-deficient cells stimulated with TLR ligands or bacteria produce an increase in NF- $\kappa$ B and MAPK activation and elevated amounts of proinflammatory cytokines, such as IL-12, IL-6, and TNF- $\alpha$ [25]. IRAK-M expression is induced upon LPS stimulation, and endotoxin tolerance is diminished in IRAK-M-deficient cells; these observations indicate that IRAK-M plays a critical role in regulating 

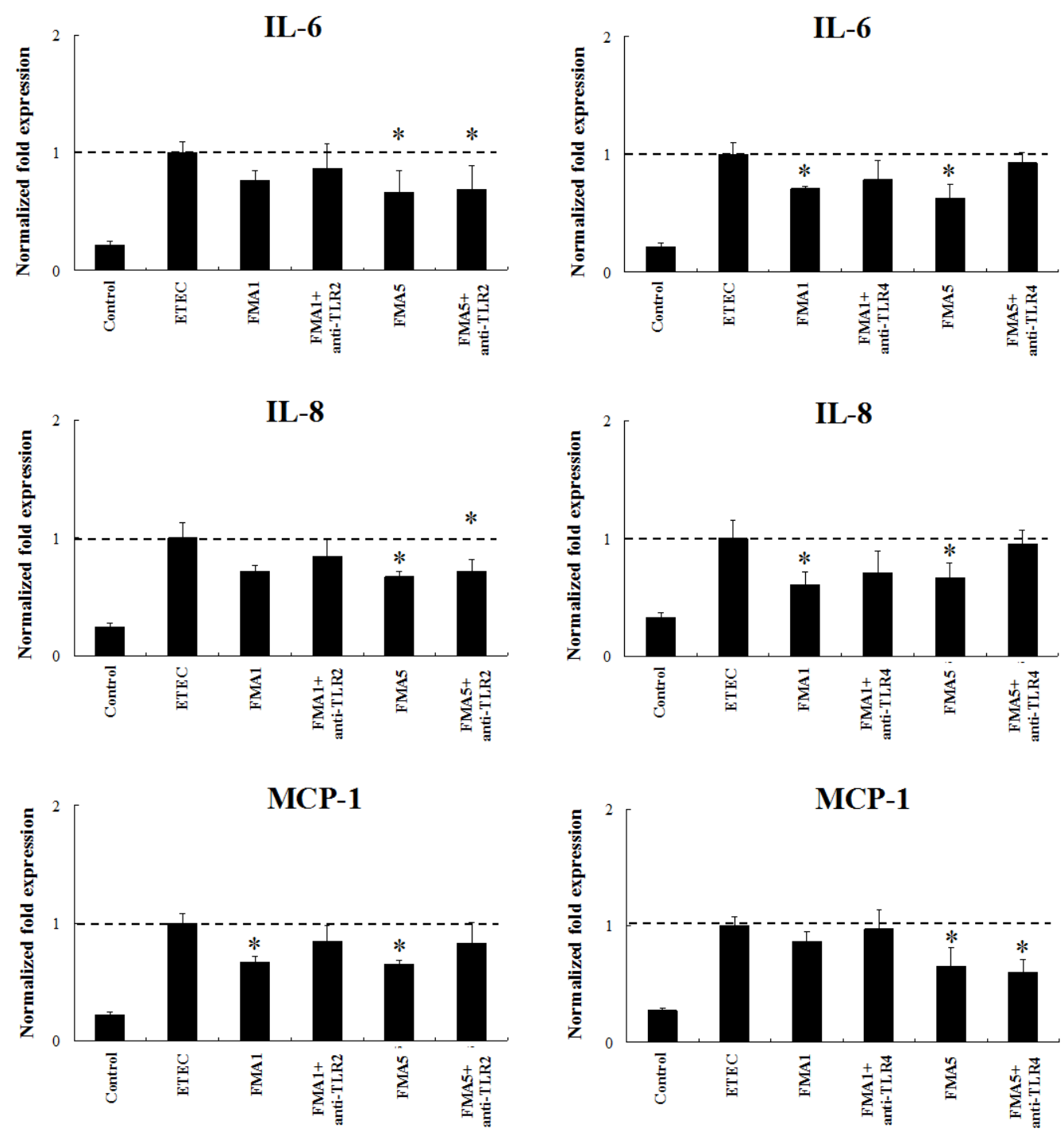

(a)

(b)

Figure 6. Role of Toll-like receptor (TLR)-2 and TLR4 s in the immunomodulatory effect of feed microbial materials (FMAs) in porcine intestinal epithelial (PIE) cells. PIE cells were pre-treated with FMAs in the presence or absence of anti-TLR2 or TLR4 antibodies. Untreated PIE cells were used as controls. PIE cells were stimulated with heat-killed enterotoxigenic Escherichia coli (ETEC) and then the expression of MCP-1, IL-6, and IL-8 mRNA were studied at $12 \mathrm{~h}$ post-stimulation. FMA1: Grinded freeze dry soymeal fermented with Enterococcus faecium and Aspergillus oryzae. FMA5: Freeze dry of Bifidobacterium thermophilum cultures. Values represent means and error bars indicate the S.D. The results are representative of three independent experiments. ${ }^{*} \mathrm{p}<0.05$ against ETEC control.

innate immunity through a negative feedback loop [26]. In addition, Tollip ensures a state of non-responsiveness in cultured enterocytes at re-exposure to LPS, due to down-regulation of TLR surface expression and decreased phosphorylation of IRAK-1. Tollip is known to be expressed at high levels in IECs, and to thereby contribute to the hyporesponsiveness of IECs to commensals [27]. Thus, the induction of these negative regulators by FMA5 in PIE cells may be important for establishing NF-kB- and p38 MAPK-mediated tolerance of ETEC.

We also demonstrated previously that $L$. jensenii TL2937, B. longum BB536 and B. breve M-16V significantly upregulated A20 in PIE cells in a TLR2-dependent manner [12] [13]. A20 plays an essential role in the termination of NF-kB signaling in response to TNF- and microbial products such as LPS [28]. Moreover, A20 deficiency in enterocytes renders mice sensitive to TNF-induced lethal inflammation, leading to disruption of the epithelial barrier and infiltration of commensal bacteria that initiate a systemic inflammatory response [29]. 
Therefore, we speculated that the strongest immunobiotic strains would induce anti-inflammatory effects in PIE cells through TLR2 and A20. We showed here that B. thermophilum present in FMA5 was able to upregulate A20 in PIE cells. Moreover, blocking experiments with anti-TLR2 antibodies significantly reduced the capacity of FMA5 to downregulate MCP-1 expression. Then, the present experiments confirm our previous results showing that A20 and TLR2 are important for the anti-inflammatory effect of immunobiotics.

In addition, TLR4 seems to have an important role in the immunoregulatory effect of FMA5 since blocking experiments with anti-TLR4 antibodies inhibit the reduction of IL-6 and IL-8 induced by FMA5. It was demonstrated that IECs shortly after birth exhibit a transient transcriptional activation induced by exposure to environmental endotoxin since it is almost completely abolished in TLR4-deficient mice [30] [31]. This transient intestinal epithelial activation precedes the acquisition of epithelial innate immune tolerance, a state of functional hyporesponsiveness to TLR4 stimuli. Epithelial tolerance is accompanied by a marked downregulation of the essential TLR signalling molecule interleukin 1 associated kinase (IRAK)-1 and a strong elevation of microRNA (miR)-146a expression [30] [31]. Moreover, epithelial tolerance appears to be maintained during the postnatal period by continuous TLR4 signalling. Further studies are necessary to establish the exact role of TLR4 in the immunoregulatory effect of FMA5. However, although the anti-inflammatory activities of probiotic microorganisms against ETEC, Salmonella Typhimurium, LPS or flagellin challenge have been reported previously using IECs cultures [20] [32], this is the first report of the immunoregulatory effect of a FMA on porcine IECs that is able to induce its effect in a TLR4-dependent manner.

\section{Conclusion}

The scientific research into probiotic mode of actions has come to age and has shown how immunobiotics are able to induce beneficial changes in the host. The study of immunobiotics-intestinal cell interactions has unraveled several molecular mechanisms and cellular pathways, showing that these interactions play a crucial role in the regulation of several immunological functions in the gut. Our present research work allows us to give a view of the cellular and molecular mechanisms involved in the immunoregulatory effects of Bifidobacterium thermophilum. These results improve the scientific basis to use this feed material to modulate intestinal epithelial and immune cells and exert beneficial effects in pigs. This feed is expected to contribute to the healthy growth of young pigs without using antimicrobial feed materials.

\section{Acknowledgements}

This study was supported by a Grant-in-Aid for Scientific Research (B) (2) (No.21380164, 24380146) and Challenging Exploratory Research (No. 23658216) from the Japan Society for the Promotion of Science (JSPS) to Dr. H. Kitazawa. Dr. Paulraj Kanmani was supported by JSPS (Postdoctoral Fellowship for Foreign Researchers, Program No. 25-03397).

\section{References}

[1] Lalles, J.P., Boudry, G., Favier, C., Le Floc’h, N., Luron, I., Montagne, L., Oswald, I.P., Pié, S. and Piel Sève, B. (2004) Gut Function and Dysfunction in Young Pigs: Physiology. Animal Research, 53, 301-316. http://dx.doi.org/10.1051/animres:2004018

[2] Williams, B.A., Verstegen, M.W.A. and Tamminga, S. (2001) Fermentation in the Large Intestine of Single-Stomached Animals and Its Relationship to Animal Health. Nutrition Research Reviews, 14, 207-227. http://dx.doi.org/10.1079/NRR200127

[3] McDermott, P.F., Walker, R.D. and White, D.G. (2003) Antimicrobials: Modes of Action and Mechanisms of Resistance. International Journal of Toxicolology, 22, 135-143.

[4] Kemper, N. (2008) Veterinary Antibiotics in the Aquatic and Terrestrial Environment. Ecological Indicators, 8, 1-13. http://dx.doi.org/10.1016/j.ecolind.2007.06.002

[5] Qiao, S., Huang, C., Lifa, D., Piao, X. and Ren, J. (2004) Effects of Lactobacilli on the Performance, Diarrhoea Incidence, VFA Concentration and Gastrointestinal Microbial Flora of 29 Weaning Pigs. Asian-Australasian. Journal of Animal Science, 17, 401-409.

[6] Herfel, T.M., Jacobi, S.K., Lin, X., Jouni, Z.E., Chichlowski, M., Stahl, C.H. and Odle, J. (2013) Dietary Supplementation of Bifidobacterium longum Strain AH1206 Increases Its Cecal Abundance and Elevates Intestinal Interleukin-10 Expression in the Neonatal Piglet. Food Chemical Toxicology, 60, 116-122. http://dx.doi.org/10.1016/j.fct.2013.07.020 
[7] Deng, J., Li, Y., Zhang, J. and Yang, Q. (2013) Co-Administration of Bacillus subtilis RJGP16 and Lactobacillus salivarius B1 Strongly Enhances the Intestinal Mucosal Immunity of Piglets. Research in Veterinary Science, 94, 62-68. http://dx.doi.org/10.1016/j.rvsc.2012.07.025

[8] Lee, J.S., Awji, E.G., Lee, S.J., Tassew, D.D., Park, Y.B., Park, K.S., Kim, M.K., Kim, B. and Park, S.C. (2012) Effect of Lactobacillus plantarum CJLP243 on the Growth Performance and Cytokine Response of Weaning Pigs Challenged with Enterotoxigenic Escherichia coli. Journal of Animal Science, 90, 3709-3717. http://dx.doi.org/10.2527/jas.2011-4434

[9] Li, X.Q., Zhu, Y.H., Zhang, H.F., Yue, Y., Cai, Z.X., Lu, Q.P., Zhang, L., Weng, X.G., Zhang, F.J., Zhou, D., Yang, J.C. and Wang, J.F. (2012) Risks Associated with High-Dose Lactobacillus rhamnosus in an Escherichia coli Model of Piglet Diarrhoea: Intestinal Microbiota and Immune Imbalances. PLOS ONE, 7, e40666. http://dx.doi.org/10.1371/journal.pone.0040666

[10] Sasaki, T., Maede, Y. and Namioka, S. (1987) Immunopotentiation of the Mucosa of the Small Intestine of Weaning Piglets by Peptidoglycan. Japanese Journal of Veterinary Science, 49, 235-243. http://dx.doi.org/10.1292/jvms1939.49.235

[11] Fujie, H., Villena, J., Tohno, M., Morie, K., Shimazu, T., Aso, H., Suda, Y., Iwabuchi, N., Xiao, J., Iwatsuki, K., Kawai, Y., Saito, T. and Kitazawa, H. (2011) Toll-Like Receptor-2 Activating Bifidobacteria Strains Differentially Regulate Inflammatory Cytokines in Porcine Intestinal Epithelial Cell Culture System: Finding New Anti-Inflammatory Immunobiotics. FEMS Immunology and Medical Microbiology, 63, 129-139. http://dx.doi.org/10.1111/j.1574-695X.2011.00837.x

[12] Shimazu, T., Villena, J., Tohno, M., Fujie, H., Hosoya, S., Shimosato, T., Aso, H., Suda, Y., Kawai, Y., Saito, T., Makino, S., Ikegami, S., Itoh, H. and Kitazawa, H. (2012) Immunobiotic Lactobacillus jensenii Elicit Anti-Inflammatory Activity in Porcine Intestinal Epithelial Cells by Modulating Negative Regulators of the Toll-Like Receptor Signaling Pathway. Infection and Immunity, 80, 276-288. http://dx.doi.org/10.1128/IAI.05729-11

[13] Tomosada, Y., Villena, J., Murata, K., Chiba, E., Shimazu, T., Aso, H., Iwabuchi, N., Xiao, J.Z., Saito, T. and Kitazawa, H. (2013) Immunoregulatory Effect of Bifidobacteria Strains in Porcine Intestinal Epithelial Cells through Modulation of Ubiquitin-Editing Enzyme A20 Expression. PLOS ONE, 8, e59259. http://dx.doi.org/10.1371/journal.pone.0059259

[14] Villena, J., Suzuki, R., Fujie, H., Chiba, E., Takahashi, T., Shimazu, T., Aso, H., Ohwada, S., Suda, Y., Ikegami, S., Itoh, H., Alvarez, S., Saito, T. and Kitazawa, H. (2012) Immunobiotic Lactobacillus jensenii Modulates Toll-Like Receptor 4-Induced Inflammatory Response via Negative Regulation in Porcine Antigen Presenting Cells. Clinical and Vaccine Immunology, 19, 1038-1053. http://dx.doi.org/10.1128/CVI.00199-12

[15] Murata, K., Tomosada, Y., Villena, J., Chiba, E., Shimazu, T., Aso, H., Iwabuchi, N., Xiao, J.Z., Saito, T. and Kitazawa, H. (2014) Bifidobacterium breve MCC-117 Induces Tolerance in Porcine Intestinal Epithelial Cells: Study of the Mechanisms Involved in the Immunoregulatory Effect. Bioscience of the Microbiota, Food and Health, 33, 1-10. http://dx.doi.org/10.12938/bmfh.33.1

[16] Moue, M., Tohno, M., Shimazu, T., Kido, T., Aso, H., Saito, T. and Kitazawa, H. (2008) Toll-Like Receptor 4 and Cytokine Expression Involved in Functional Immune Response in an Originally Established Porcine Intestinal Epitheliocyte Cell Line. Biochimeca and Biophysica Acta, 1780, 134-144.

[17] Lalles, J.P., Bosia, P., Smidta, H. and Stokes, C.R. (2007) Nutritional Management of Gut Health in Pigs around Weaning. Proceedings of the Nutrition Society, 66, 260-268. http://dx.doi.org/10.1017/S0029665107005484

[18] Salva, S., Villena, J. and Alvarez, S. (2010) Differential Immunomodulatory Activity of Lactobacillus rhamnosus Strains Isolated from Goat Milk: Impact on Intestinal and Respiratory Infections. International Journal of Food Microbiology, 141, 82-89. http://dx.doi.org/10.1016/j.ijfoodmicro.2010.03.013

[19] Chiba, E., Tomosada, Y., Vizoso-Pinto, M.G., Takahashi, T., Tsukida, K., Kitazawa, H., Avarez, S. and Villena, J. (2013) Immunobiotic Lactobacillus rhamnosus Improves Resistance of Infant Mice against Respiratory Syncytial Virus Infection. International Immunopharmacolology, 17, 373-382. http://dx.doi.org/10.1016/j.intimp.2013.06.024

[20] Roselli, M., Finamore, A., Britti, M.S. and Mengheri, E. (2006) Probiotic Bacteria Bifidobacterium animalis MB5 and Lactobacillus rhamnosus GG Protect Intestinal Caco-2 Cells from the Inflammation-Associated Response Induced by Enterotoxigenic Escherichia coli K88. British Journal of Nutrition, 95, 1177-1184. http://dx.doi.org/10.1079/BJN20051681

[21] Roselli, M., Finamore, A., Britti, M.S., Konstantinov, S.R., Smidt, H., de Vos, W.M. and Mengheri, E. (2007) The Novel Porcine Lactobacillus sobrius Strain Protects Intestinal Cells from Enterotoxigenic Escherichia coli K88 Infection and Prevents Membrane Barrier Damage. Journal of Nutrition, 137, 2709-2716.

[22] O’Hara, A.M., O’Regan, P., Fanning, A., O’Mahony, C., Macsharry, J., Lyons, A., Bienenstock, J., O’Mahony, L. and Shanahan, F. (2006) Functional Modulation of Human Intestinal Epithelial Cell Responses by Bifidobacterium infantis and Lactobacillus salivarius. Immunology, 118, 202-215. http://dx.doi.org/10.1111/j.1365-2567.2006.02358.x 
[23] Qin, J., Qian, Y., Yao, J., Grace, C. and Li, X. (2005) SIGIRR Inhibits Interleukin-1 Receptor- and Toll-Like Receptor 4-Mediated Signaling through Different Mechanisms. Journal of Biological Chemistry, 280, 25233-25241. http://dx.doi.org/10.1074/jbc.M501363200

[24] Lech Garlanda, M., Mantovani, C., Kirschning, A., Schlöndorff, C.J. and Anders, D. (2007) Different Roles of TiR8/Sigirr on Toll-Like Receptor Signaling in Intrarenal Antigen-Presenting Cells and Tubular Epithelial Cells. Kidney International, 72, 182-192. http://dx.doi.org/10.1038/sj.ki.5002293

[25] Deng, J.C., Cheng, G., Newstead, M.W., Zeng, X., Kobayashi, K., Flavell, R.A. and Standiford, T.J. (2006) Sepsis-Induced Suppression of Lung Innate Immunity Is Mediated by IRAK-M. Journal of Clinical Investigation, 116, 2532-2542.

[26] Escoll, P., del Fresno, C., García, L., Vallés, G., Lendínez, M.J., Arnalich, F. and López-Collazo, E. (2003) Rapid Up-Regulation of IRAK-M Expression Following a Second Endotoxin Challenge in Human Monocytes and in Monocytes Isolated from Septic Patients. Biochemical and Biophysical Research Communications, 311, 465-472. http://dx.doi.org/10.1016/j.bbrc.2003.10.019

[27] Otte, J.M., Cario, E. and Podolsky, D.K. (2004) Mechanisms of Cross Hyporesponsiveness to Toll-Like Receptor Bacterial Ligands in Intestinal Epithelial Cells. Gastroenterology, 126, 1054-1070. http://dx.doi.org/10.1053/j.gastro.2004.01.007

[28] Wang, J., Ouyang, Y., Guner, Y., Ford, H.R. and Grishin, A.V. (2009) Ubiquitin-Editing Enzyme A20 Promotes Tolerance to Lipopolysaccharide in Enterocytes. Journal of Immunology, 183, 1384-1392. http://dx.doi.org/10.4049/jimmunol.0803987

[29] Lee, E.G., Boone, D.L., Chai, S., Libby, S.L. and Chien, M. (2000) Failure to Regulate TNF-Induced NF-kB and Cell Death Responses in A20-Deficient Mice. Science, 289, 2350-2354. http://dx.doi.org/10.1126/science.289.5488.2350

[30] Lotz, M., Gutle, D., Walther, S., Menard, S., Bogdan, C. and Hornef, M.W. (2006) Postnatal Acquisition of Endotoxin Tolerance in Intestinal Epithelial Cells. The Journal of Experimental Medicine, 203, 973-984. http://dx.doi.org/10.1084/jem.20050625

[31] Chassin, C., Kocur, M., Pott, J., Duerr, C.U., Gutle, D. and Lotz, M. (2010) miR-146a Mediates Protective Innate Immune Tolerance in the Neonate Intestine. Cell Host and Microbe, 8, 358-368. http://dx.doi.org/10.1016/j.chom.2010.09.005

[32] Riedel, C.U., Foata, F., Philippe, D., Adolfsson, O., Eikmanns, B.J. and Blum, S. (2006) Anti-Inflammatory Effects of Bifidobacteria by Inhibition of LPS-Induced NF-kappaB Activation. World Journal of Gastroenterology, 12, 37293735.

\section{List of Abbreviations}

Enterotoxigenic escherichia coli (ETEC); functional feed materials (FMAs); intestinal epithelial cells (IECs); mesenteric lymph nodes (MLNs); Peyer's patches (PPs); porcine intestinal epithelial (PIE); Toll-like receptor (TLR) 